\title{
A procedure for evaluating portable heart monitors
}

\author{
DAVID G. BORN, JOHN ARAUJO, and TOM R. THOMAS \\ University of Kansas, Lawrence, Kansas
}

\begin{abstract}
Portable heart rate monitors are potentially useful for regulating exercise intensity. This paper describes procedures to evaluate these devices while subjects are resting, cycling, or running. Of the five monitors tested, all performed reasonably well while subjects were at rest; however, with one exception, performance deteriorated when the monitors were used during exercise. Even so, depending on the level of accuracy required, some of the monitors remained useful during cycling, but only one monitor was consistently accurate while subjects were running. This evidence indicates that, before selecting a particular monitor, potential users of such equipment should carefully consider many things, including the circumstances under which the equipment will be employed. The procedures reported in this paper should be generally useful in evaluating the performance of a variety of devices for monitoring heart rate.
\end{abstract}

Frequently, a measure of heart rate is used for controlling the intensity of exercise. Although the carotid artery is probably the easiest artery to palpate, this technique does not always yield accurate results (White, 1977). Palpation of the radial artery can be accurate, but finding a pulse there is often difficult, especially in older adults, and palpation may be unreliable at high heart rates or while exercise is ongoing.

Although radiotelemetry and electrocardiograph (ECG) monitoring have been utilized to accurately determine exercise heart rate, these techniques are expensive and not always practical. Recently, portable electronic heart monitors have become available. Although these devices hold much promise for ascertaining heart rate, they are also expensive and must offer clear advantages over artery or chest palpation to be worthwhile. A brief report ("These four gadgets," 1980) suggested that at least some portable monitors do not yield stable results during exercise. This paper reports a more extensive evalutation of five portable heart monitors under conditions of rest (i.e., standing still) and during two modes of exercise, running and cycling. More importantly, this paper describes a methodology for evaluating new entries into this rapidly expanding field.

\section{METHOD}

\section{Equipment ${ }^{1}$}

Five portable heart monitors were evaluated. The major characteristics of each of these units are summarized in Table 1.

Two of the units, EXERSENTRY and INSTA PULSE, use the ECG signal to monitor heart rate. EXERSENTRY is held in

D. G. Born and J. Ataujo are with the Department of Human Development, and T. R. Thomas is with the Department of Health, Physical Education, and Recreation. Requests for reprints should be sent to David G. Born, Department of Human Development, University of Kansas, Lawrence, KS 66045. direct contact with the chest by an elastic harness that positions three ECG electrodes. In addition to heart rates, this monitor also provides the user with two different audio signals, which sound when heart rate falls below or above a user-specified limit [range $=35-220$ beats per minute $(\mathrm{bpm})]$. INSTA PULSE is similar in size and shape to a track-and-field baton. Its LED indicator is activated by touching the rings at each end-normally accomplished by holding one hand on each end.

The remaining three instruments, APOLLO, HEARTWATCH, and POCKET PULSE MINDER, monitor heart rate with a finger-optic plethysmograph that detects the change in reflected light (produced by pulsating blood) associated with each pulse. With APOLLO, the user has a choice of two different fingeroptic pickups. With one pickup, a finger is inserted into a springloaded clip containing both a light source and a sensor. The other pickup option appears as a notch on the side of the unit. With light, firm pressure, the user covers this notch with the index finger of the right hand. HEARTWATCH is physically similar to APOLLO, but has two major differences: (1) A switch permits a user to select heart rate averaging based on either four (HEARTWATCH-4) or eight (HEARTWATCH-8) heartbeats, and (2) there is only a clip for detecting finger pulse. Due to its size and shape, the POCKET PULSE MINDER is intended primarily for stationary exercise. Heart rate is indicated by the position of a needle on a calibrated heart rate dial. In addition, there is the choice of a red light that flashes or an audio signal that beeps with each heartbeat.

Except for INSTA PULSE and POCKET PULSE MINDER, the instruments described here take a sample of heartbeats (usually four) and then compute and display a heart rate. POCKET PULSE MINDER uses an analog averaging process and updates the calculated heart rate with each succeeding beat. INSTA PULSE computes and displays heart rate based on each interheartbeat interval, thereby displaying a new heart rate after each heartbeat.

\section{Subjects}

Three men and three women, between the ages of 21 and 28 years and all in very good physical condition, operated each of the portable monitors under three steady-state test conditionsat rest, during treadmill running, and during stationary cycling.

\section{Test Conditions}

During the resting condition, heart rate data were collected while the subject stood quietly on the treadmill. 
Table 1

Characteristics of the Five Portable Heart Rate Monitors

\begin{tabular}{llllll}
\multicolumn{1}{c}{ Features } & $\begin{array}{l}\text { EXER- } \\
\text { SENTRY }\end{array}$ & $\begin{array}{c}\text { INSTA } \\
\text { PULSE }\end{array}$ & APOLLO & $\begin{array}{c}\text { HEART } \\
\text { WATCH }\end{array}$ & $\begin{array}{c}\text { POCKET } \\
\text { PULSE } \\
\text { MINDER }\end{array}$ \\
\hline Retail Price & $\$ 339.00$ & $\$ 156.00$ & $\$ 129.00$ & $\$ 189.00$ & $\$ 193.00$ \\
Warranty & 1 year & 90 days & none & 1 year & 1 year \\
Indicator & LCD & LED & LCD & LCD & Meter \\
Battery Type & NR & NR & R & R & NR \\
Hours of Continuous Operation & $200-300$ & $25-30$ & NP & 2 & NP \\
Range (bpm) & $35-220$ & $0-999$ & $40-200$ & NP & $0-200$ \\
\hline
\end{tabular}

Note $-N R=$ nonrechargeable; $R=$ rechargeable (chargers are included in the price of APOLLO and HEARTWATCH; their respective charging times are $12-18$ and $4 \mathrm{~h}$ ). $N P=$ not provided. $b p m=$ beats per minute.

For the treadmill-running condition, the treadmill speed (0\% incline) was adjusted to provide an intensity that subjects could comfortably maintain for 6 to $8 \mathrm{~min}$, and data were collected when the heart rate was stable at some point above 130 bpin but below 170 bpm.

For stationary cycling, the subjects pedaled at 50 revolutions per minute in cadence with an electric metronome. The cycle load was adjusted to provide an exercise intensity meeting the criterion used during treadmill running. Since two hands were required for operation of the INSTA PULSE, it was not evaluated during cycle exercise.

\section{Test Protocol}

Each unit, containing either fresh or fully charged batteries, was evaluated for $30 \mathrm{sec}$ in each condition by recording the heart rate values displayed as they were called out by the subject. These values were recorded on audio tape (providing a method for later verification) and also were transcribed by an assistant. A Quinton ECG system, with modified Lead II electrode placement, was used to monitor heart rates for comparison with the portable monitors.

Two of the monitors forced a minor modification of the above procedures. Because POCKET PULSE MINDER has a continuous needle/dial heart rate display, there is no discrete updating of heart rate for a subject to report. With INSTA PULSE, during treadmill testing and stationary cycling (when heart rates were elevated), it was impossible for a subject to call out each display, because the numbers were changing too rapidly. Therefore, data were obtained from each of these instruments in the following manner. An observer, monitoring the Quinton oscilloscope, counted out heartbeats as they appeared on the oscilloscope screen. When the fourth beat in the series had been signaled by the observer, the subject called out the number displayed by the heart monitor at that time. In this manner, the heart rate at every fourth heartbeat was recorded. ${ }^{2}$

\section{Computing Heart Monitor Accuracy}

The number of heartbeats from each 30-sec ICC strip chart was doubled to obtain the number of heartbeats per minutc in each test condition for each subject. These were the values subsequently compared with comparable averages from the portable heart monitors.

The written record of heart rates from each portable monitor was first verified with the audio tapes of the test sessions. ${ }^{3}$ These data were then scrutinized for scores that were either obviously inaccurate (e.g., heart rates greater than $200 \mathrm{bpm}$ ) or were strikingly inconsistent with other values from the test period. Suspect scores were then assessed with Dixon's (1953) test of extreme scores and were deleted when appropriate. Finally, a mean and standard deviation were computed from the remaining series of heart rates.

Since the results frequently revealed wide variations in instrument functioning between subjects, the decision was made to present the data in a manner that preserved both individual values and exercise conditions.
To arrive at an "accuracy" value for each monitor, the heart rate derived from the Quinton ECG strip chart was subtracted from the corresponding mean of the values obtained from each portable monitor. The results of this comparison appear in Table 2. The number of "extreme" scores deleted from each test condition is preserved through the use of superscripts. For example, Table 2 shows that for Subject $A$ there was a discrepancy of $5 \mathrm{bpm}$ between the mean heart rate produced by the APOLLO instrument with finger clip and the ECG record; the superscript on the number 5 (i.e., $5^{1}$ ) indicates that one score was deleted from the series generated during this particular assessment interval.

Table 3 shows the range and standard deviation of the series of heart rate values. As in Table 2, a superscript in Table 3 also indicates the number of extreme scores deleted from the series.

\section{RESULTS}

\section{Resting Heart Rates}

ECG monitors. Both the EXERSENTRY and INSTA PULSE performed well during the resting heart rate evaluation. In the case of EXERSENTRY, five of the six mean readings were within $1 \mathrm{bpm}$ of their corresponding ECG records. With the INSTA PULSE, the mean readings were within $2.7 \mathrm{bpm}$ of the ECG records.

Finger plethysmographs. In general, the equipment with finger plethysmographs performed reasonably well when the subjects were inactive. The accuracy scores in Table 2 show that, with Subject A, for example, the average of the displays produced by HEARTWATCH-4 over the 30 -sec test interval was only $2 \mathrm{bpm}$ higher than the ECG record for the same period. In fact, HEARTWATCH-4 was within $2 \mathrm{bpm}$ for all subjects, and HEARTWATCH-8 was even better.

Although the APOLLO instrument worked well for most subjects, there were two notable exceptions. Even with a concerted effort to select a finger and a position for the finger clip that would result in acceptable readings, Subject $C$ was unable to get this instrument to produce reasonable heart rate values. The result was an average discrepancy of $-56 \mathrm{bpm}$. A similar problem was encountered with Subject $E$ during the assessment of the APOLLO's side pickup. In this case, the instrument indicated a heart rate that was $31 \mathrm{bpm}$ lower than the ECG.

The POCKET PULSE MINDER readings deviated only a few beats per minute from the ECG record, with 
Table 2

Heart Rate Discrepancies

\begin{tabular}{|c|c|c|c|c|c|c|c|}
\hline \multirow[b]{2}{*}{ Subject } & \multicolumn{2}{|c|}{ ECG Monitors } & \multicolumn{5}{|c|}{ Finger Optic Plethysmographs } \\
\hline & $\begin{array}{l}\text { EXER- } \\
\text { SENTRY }\end{array}$ & $\begin{array}{l}\text { INSTA } \\
\text { PULSE }\end{array}$ & $\begin{array}{l}\text { APOLLO/ } \\
\text { CLIP }\end{array}$ & $\begin{array}{l}\text { APOLLO/ } \\
\text { SIDE }\end{array}$ & $\mathrm{HW}-4$ & HW-8 & $\begin{array}{c}\text { POCKET } \\
\text { PULSE } \\
\text { MINDER }\end{array}$ \\
\hline \multicolumn{8}{|c|}{ At Rest } \\
\hline $\mathbf{A}$ & 1 & $4^{1}$ & $5^{1}$ & 1 & 2 & 1 & $8^{1}$ \\
\hline B & -1 & 3 & $-1^{1}$ & -1 & -2 & 0 & 0 \\
\hline $\mathrm{C}$ & 1 & 2 & 56 & -4 & 0 & -1 & -1 \\
\hline $\mathrm{D}$ & -1 & 7 & -1 & -1 & -1 & -1 & -3 \\
\hline $\mathrm{E}$ & 0 & 4 & 1 & -31 & 2 & 0 & -1 \\
\hline $\mathrm{F}$ & 3 & $3^{1}$ & 0 & 1 & 1 & 0 & -2 \\
\hline \multicolumn{8}{|c|}{ Running } \\
\hline A & 1 & $16^{7}$ & -6 & -59 & -27 & -9 & -8 \\
\hline B & 0 & 11 & -24 & $-6^{2}$ & -31 & $-33^{1}$ & $*$ \\
\hline $\mathrm{C}$ & 0 & $13^{1}$ & $-35^{5}$ & $-33^{3}$ & -11 & -22 & 37 \\
\hline $\mathrm{D}$ & 0 & $2^{8}$ & -55 & -58 & -14 & -17 & -5 \\
\hline $\mathrm{E}$ & 1 & $11^{2}$ & -39 & -35 & -40 & -26 & -34 \\
\hline $\mathrm{F}$ & -4 & $-67^{7}$ & -26 & -72 & -5 & -22 & -15 \\
\hline \multicolumn{8}{|c|}{ Cycling } \\
\hline A & 0 & & -4 & -32 & -1 & 0 & -14 \\
\hline B & 0 & & -26 & -70 & 12 & -44 & -12 \\
\hline $\mathrm{C}$ & 0 & & $0^{4}$ & -63 & -2 & -1 & -6 \\
\hline D & -2 & & -3 & -38 & $1^{1}$ & -2 & 16 \\
\hline E & 1 & & 0 & -85 & 1 & 0 & -9 \\
\hline $\mathrm{F}$ & 0 & & 2 & -84 & -1 & -2 & -42 \\
\hline
\end{tabular}

Note-Each cell contains (1) the mean of the heart rate displays minus the ECG determined heart rate, and (2) a superscript indicating the number of extreme scores deleted. When a number in a cell does not carry a superscript, it means score deletion was unnecessary. INSTA PULSE was not used during the cycle condition-see the Method section. *No data were collected from Subject B during the treadmill test with the POCKET PULSE MINDER.

Table 3

Variability in Heart Rate Displays

\begin{tabular}{|c|c|c|c|c|c|c|c|c|c|c|c|c|c|c|}
\hline \multirow[b]{3}{*}{ Subject } & \multicolumn{4}{|c|}{ ECG Monitors } & \multicolumn{10}{|c|}{ Finger Optic Plethysmographs } \\
\hline & \multicolumn{2}{|c|}{$\begin{array}{c}\text { EXER- } \\
\text { SENTRY }\end{array}$} & \multicolumn{2}{|c|}{$\begin{array}{l}\text { INSTA } \\
\text { PULSE }\end{array}$} & \multicolumn{2}{|c|}{$\begin{array}{l}\text { APOLLO/ } \\
\text { CLIP }\end{array}$} & \multicolumn{2}{|c|}{$\begin{array}{l}\text { APOLLO/ } \\
\text { SIDE }\end{array}$} & \multicolumn{2}{|c|}{$\mathrm{HW}-4$} & \multicolumn{2}{|c|}{ HW-8 } & \multicolumn{2}{|c|}{$\begin{array}{l}\text { POCKET } \\
\text { PULSE } \\
\text { MINDER }\end{array}$} \\
\hline & SD & $\mathbf{R}$ & SD & $\mathbf{R}$ & SD & $\mathbf{R}$ & SD & $\mathbf{R}$ & SD & $\mathbf{R}$ & SD & $\mathbf{R}$ & SD & $\mathbf{R}$ \\
\hline \multicolumn{15}{|c|}{ At Rest } \\
\hline A & 3.3 & 11 & $3.1^{1}$ & $10^{1}$ & $9.5^{1}$ & $27^{1}$ & 10.3 & 37 & 5.3 & 16 & 1.7 & 3 & $11.1^{1}$ & $30^{1}$ \\
\hline B & 1.4 & 4 & 4.1 & 11 & $1.0^{3}$ & $3^{1}$ & 0.5 & 1 & 3.0 & 8 & 2.1 & 4 & 2.4 & 5 \\
\hline $\mathrm{C}$ & 3.5 & 12 & 4.2 & 14 & 28.6 & 97 & 9.5 & 25 & 4.2 & 13 & 2.2 & 6 & 2.5 & 7 \\
\hline $\mathrm{D}$ & 1.9 & 5 & 5.2 & 21 & $\begin{array}{r}20.0 \\
4.2\end{array}$ & 14 & 7.7 & 29 & 3.1 & 10 & $\begin{array}{l}2.2 \\
3.6\end{array}$ & 8 & $\begin{array}{l}2.3 \\
1.6\end{array}$ & 4 \\
\hline $\mathbf{E}$ & 1.9 & 6 & 3.8 & 12 & 2.1 & 7 & 4.2 & 6 & 5.1 & 17 & 2.6 & 7 & 3.2 & 10 \\
\hline $\mathrm{F}$ & 7.1 & 22 & $5.1^{1}$ & $12^{1}$ & 4.4 & 17 & 6.9 & 21 & 2.9 & 9 & 3.5 & 9 & 2.4 & 7 \\
\hline \multicolumn{15}{|c|}{ Running } \\
\hline $\mathbf{A}$ & 1.4 & 5 & $12.9^{7}$ & $29^{7}$ & 10.6 & 28 & 14.5 & 30 & 16.7 & 58 & 10.3 & 28 & 4.2 & 10 \\
\hline B & 1.0 & 5 & 7.4 & 29 & 22.7 & 67 & $34.2^{2}$ & $100^{2}$ & 16.8 & 50 & $7.2^{1}$ & $19^{2}$ & $*$ & $*$ \\
\hline $\mathrm{C}$ & 0.9 & 3 & $12.6^{1}$ & $44^{1}$ & $8.6^{5}$ & $22^{5}$ & $24.5^{3}$ & $98^{3}$ & 14.4 & 40 & 10.0 & 33 & 8.0 & 22 \\
\hline $\mathrm{D}$ & 1.9 & $\begin{array}{l}5 \\
6\end{array}$ & $\begin{array}{l}12.0 \\
18.6^{8}\end{array}$ & $\begin{array}{l}44^{8} \\
60^{8}\end{array}$ & $\begin{array}{l}0.0 \\
13.0\end{array}$ & $\begin{array}{l}22 \\
46\end{array}$ & $\begin{array}{l}24.3 \\
27.2\end{array}$ & 109 & $\begin{array}{l}14.4 \\
16.7\end{array}$ & $\begin{array}{l}40 \\
50\end{array}$ & $\begin{array}{l}10.0 \\
16.7\end{array}$ & $\begin{array}{l}33 \\
44\end{array}$ & $\begin{array}{l}8.0 \\
3.6\end{array}$ & $\begin{array}{l}22 \\
12\end{array}$ \\
\hline $\mathrm{E}$ & 1.1 & 3 & $9.0^{2}$ & $31^{2}$ & 21.2 & 60 & 32.3 & 102 & 17.9 & 63 & 15.6 & 46 & 9.0 & 32 \\
\hline$F$ & 4.0 & 12 & $31.7^{7}$ & $102^{7}$ & 32.6 & 85 & 18.7 & 63 & 13.6 & 41 & $\begin{array}{l}10.0 \\
19.9\end{array}$ & $\begin{array}{l}40 \\
65\end{array}$ & $\begin{array}{l}7.0 \\
8.6\end{array}$ & 25 \\
\hline \multicolumn{15}{|c|}{ Cycling } \\
\hline $\mathbf{A}$ & 1.2 & 3 & & & 9.0 & 29 & 19.0 & 58 & 1.7 & 5 & 0.8 & 2 & 6.2 & 26 \\
\hline B & 0.9 & 3 & & & 15.9 & 54 & 1.5 & 4 & 13.9 & 42 & 10.8 & 27 & 7.3 & 21 \\
\hline $\mathrm{C}$ & 0.9 & 3 & & & $3.4^{4}$ & $10^{4}$ & 17.4 & 61 & 1.6 & 5 & 0.9 & 3 & 1.8 & 5 \\
\hline D & 1.2 & 4 & & & 21.9 & 63 & 15.0 & 61 & $2.9^{1}$ & $11^{1}$ & 5.5 & 18 & 4.0 & 12 \\
\hline $\mathbf{E}$ & 0.7 & 2 & & & 1.5 & 6 & 8.9 & 22 & 1.4 & 4 & 1.4 & 4 & 8.1 & 22 \\
\hline $\mathbf{F}$ & 1.8 & 5 & & & 6.1 & 22 & 5.8 & 16 & 0.8 & 3 & 0.9 & 2 & 14.4 & 45 \\
\hline
\end{tabular}

Note-SD = standard deviation; $R=$ range. $A$ superscript indicates the number of extreme scores deleted. When a number does not carry a superscript, it means no scores were deleted. INSTA PULSE was not used during the cycle condition-see Method section. *No data were collected from Subject $B$ during the treadmill test with the POCKET PULSE MINDER. 
the single exception of Subject A. In that case, the instrument indicated a rate approximately $8 \mathrm{bpm}$ too high.

\section{Treadmill Running}

ECG monitors. As with the resting heart rate assessment, EXERSENTRY averaged within $1 \mathrm{bpm}$ of the ECG record for five of the six subjects; for the remaining subject, there was a discrepancy of $4 \mathrm{bpm}$. In the case of the INSTA PULSE, the average readings tended to be considerably discrepant from the ECG records. These discrepancies ranged from $2 \mathrm{bpm}$ for Subject $D$ to $-67 \mathrm{bpm}$ for Subject F.

Finger plethysmographs. None of the finger plethysmographic instruments performed well during the treadmill tests. In fact, the average reading from one of the instruments was $72 \mathrm{bpm}$ lower than the average from the ECG over the same period. Furthermore, with one exception, all the discrepant readings were on the low side (i.e., the instrument erred on the side of displaying heart rates that were lower than those obtained from the Quinton ECG).

\section{Cycling}

ECG monitors. As with earlier assessments, the EXERSENTRY agreed very closely with the Quinton ECG records. The maximal accuracy-score disagreement across the six subjects was a heart rate underestimate of 2 bpm.

Finger plethysmographs. With exceptions in the case of each instrument, the correspondence between the Quinton ECG and displays of heart rate from the monitors during cycling was better than that during running. Both HEARTWATCH-4 and HEARTWATCH-8 averaged within $2 \mathrm{bpm}$ of the ECG for all but Subject B; in that case, HEARTWATCH-4 was $12 \mathrm{bpm}$ too high, and HEARTWATCH-8 was $44 \mathrm{bpm}$ too low. Similar anomalous readings were obtained from Subject B with the APOLLO instrument; when the finger clip was used, the average of the APOLLO readings was $26 \mathrm{bpm}$ too low, although the same instrument was reasonably accurate with the other subjects. However, when the side pickup on the APOLLO was used during cycling, the most discrepant records encountered at any time during this assessment were obtained. For unexplained reasons, the obtained heart rate readings were between 32 and $85 \mathrm{bpm}$ too low! Finally, there was substantial variability in the accuracy of the POCKET PULSE MINDER across subjects, as shown in Table 2 . In this case, the discrepancies ranged from an overestimate of $16 \mathrm{bpm}$ (Subject D) to an underestimate of $42 \mathrm{bpm}$ (Subject F).

\section{Variability}

In general, small accuracy discrepancies for a given instrument and subject tended to be associated with little variability, even though, as described previously, extreme scores were removed from the sets of measures before the summary statistics in Tables 2 and 3 were calculated.

Table 3 indicates that variability tended to be low for all instruments during the assessment of resting heart rate. However, during the treadmill test, there were substantial differences in instrument variability. On the one hand, EXERSENTRY performed exceptionally well during treadmill testing; surprisingly, both its variability and accuracy were at least as good in the treadmill condition as in the resting condition. On the other hand, the other monitors all showed considerably increased variability during treadmill testing. With the cycle ergometer test, in which there is usually less upper body movement than during running but more than during resting, levels of variability tended to be intermediate between those occurring in the other two testing conditions.

\section{DISCUSSION}

A decision to purchase portable heart monitors for clinical or research purposes depends upon many factors. All of the monitors are fairly expensive, and some will be difficult to use with particular forms of activity. In addition, there are important differences in how well the instruments function when used in various exercise modes.

In the present tests, EXERSENTRY was the only instrument that was consistently "accurate" during all three test conditions. Thus, for running, EXERSENTRY appears to be the instrument of choice. However, for a different mode of exercise, some of the other monitors might be equally useful and even preferable because of other features.

Among the finger-optic plethysmographs, HEARTWATCH seemed to be the most desirable unit. The larger physical size of the POCKET PULSE MINDER limits its application to stationary exercise, and some of our subjects complained that the meter on this instrument was difficult to read. In the case of APOLLO, finger position on the side pickup or in the sensing clip seemed to be extremely critical, and the heart rate displays were often erratic. In contrast, HEARTWATCH performed well in both the resting and the cycling conditions (with the exception of Subject B), and it may be easily carried.

In many respects, variability in measures of heart rate is as important as average accuracy. Unfortunately, the information collected in this investigation does not provide a separate and unequivocal assessment of instrument variability. During any individual test session, it was possible for the actual heart rate of a subject to change. However, as described previously, this type of variability was minimized by using steady-state test conditions, trained subjects, and short $(30-\mathrm{sec})$ test intervals. Even so, most of the instruments showed much greater variability in their heart rate displays than one would expect from our test conditions. Although some 
of this variability could have arisen from an atypical heartbeat or two (these instruments typically calculate heart rate on the basis of a very small sample of heart beats), it more likely had other origins-possibly movement of the signal pickup device.

A common reason for purchasing a heart monitor is an interest in accurately monitoring heart rate during exercise. Although only EXERSENTRY was accurate during running, some of the other monitors might still be useful if exercise were interrupted long enough to get an accurate reading. But when the monitors are first switched on, there is a short latency before the first accurate heart rate display appears.

To obtain an estimate of the latency with which APOLLO, INSTA PULSE, and HEARTWATCH were capable of producing accurate readings of resting heart rate, the interval between switching on of each instrument and the appearance of the first display that was within $\pm 10 \mathrm{bpm}$ of the current heart rate was timed. At room temperature $\left(21^{\circ} \mathrm{C}\right)$, the results of this simple test revealed little difference among the four-beat averaging monitors; they required approximately $8-10 \mathrm{sec}$ to begin producing accurate numbers. However, the INSTA PULSE required an average of slightly less than $3 \mathrm{sec}$ to become fully operational (i.e., accurate within $\pm 10 \mathrm{bpm}$ ). As one might expect, when the HEARTWATCH was set to eight-beat averaging, it had the longest latency and averaged approximately $12 \mathrm{sec}$ to become operational. Finally, because long-distance runners might expose these instruments to cold weather, the units were evaluated after being refrigerated at $0^{\circ} \mathrm{C}$. At this cooler temperature, there were no appreciable changes in operating latencies.

Although we have attempted to identify some of the relevant considerations in selecting among the available heart monitors, several factors have not been mentioned. For example, we do not know how finger-optic plethysmographs work on individuals who have circulation problems or thickly calloused hands. Also, EXERSENTRY may not work well with an obese individual, although it was accurate with Subject B, who had a very hairy chest. These few examples suggest that the physical condition of the intended user, modes of exercise, operating characteristics of the instruments, and plans for use of a monitor should all be carefully reviewed in making a prudent selection of equipment.

\section{REFERENCES}

Dixon, W. J. (1953). Processing data for outliers. Biometrics, 9, 74-89.
These Four gadgets (THROB) MEASURE heART RATE (THROB). (1980, January). Consumer Reports, p. 5.

WHITE, J. R. (1977). EKG changes using carotid artery for heart rate monitoring. Medicine and Science in Sports, 9, 86-94.

\section{NOTES}

1. The authors express their appreciation to the equipment manufacturers for providing the sample units used in this evaluation. Specifically, (1) EXERSENTRY (Model EX-3) is manufactured by Respironics, Inc., 650 Seco Road, Monroeville, PA 15146 [(412) 373-8814], (2) INSTA PULSE is manufactured by BioSig, 5471 Royalmount Avenue, Montreal, Quebec H4P 1J3, Canada [(514) 733-3362], (3) APOLLO Heart Rate Indicator is manufactured by Apollo Products, Inc., W. 10 Mission, Spokane, WA 99201 [(509) 328-5591], (4) HEARTWATCH (Model 48) is manufactured by Dart Medical Equipment, Inc., 113 E. Allegan Street, Lansing, MI 48933 [(517) 676-5533], and (5) POCKET PULSE MINDER (Model 7719) is manufactured by Computer Instruments, Inc., $100 \mathrm{Mad}-$ ison Avenue, Hempstead, NY 11550 [(516) 483-8200].

Since only a single unit from each manufacturer was evaluated, it is, of course, possible that the results obtained in our tests of a particular instrument have limited generality.

2. Because the INSTA PULSE determines heart rate from single interbeat intervals, instead of using some form of averaging, like the other monitors tested, it is difficult to compare the unit directly with the other instruments. With the averaging monitors, information from every heartbeat during the test sessions contributed to the assessment of the instruments. With INSTA PULSE, however, the data-collection procedures used information from only every fourth heartbeat. This was necessary because, especially during exercise, the INSTA PULSE displays changed so rapidly that the subjects were unable to report them all. Our procedures produced about the same number of pieces of data from each instrument, but these data were derived from individual beats, in one case, and were averages of several beats, in the other cases.

3 . Since only changes in the displayed heart rate were called out by the subjects, a test session could yield a very small number of scores from a monitor if a subject's heart rate remained very stable. To verify that the total number of scores for each session was correct, the following steps were taken. (1) The number of displays expected was obtained by dividing the number of beats on the $30-\mathrm{sec}$ strip by 4 (or 8 in the case of HEARTWATCH-8), and this number of displays was compared with that actually obtained. (2) If the two figures did not disagree by more than 1 , then the extreme-score analyses were performed. (3) If the disagreement was greater than 1 , then the average interdisplay interval was computed, and, upon replay (via the tape recorder) of the data, the appropriate number of displays (and their values) was determined. In other words, if the display changed after every four beats, then the interdisplay intervals would be approximately the same. However, when two succeeding groups of four heartbeats produced the same heart rate, then the display did not change, because there were two consecutive displays of the same value, and, thus, the interval would be twice as long. From these interdisplay-interval computations, the appropriate number of displays was determined.

(Manuscript received August 19, 1983; revision accepted for publication February 17, 1984.) 